\title{
Value of wearing head protection gear while playing hurling
}

\author{
P J Crowley*, M J Crowley ${ }^{\dagger}$, H Dardouri ${ }^{\ddagger}$ and K C Condon ${ }^{\S}$
}

${ }^{*}$ General Practitioner, West Village, Ballincollig; ${ }^{\dagger}$ Head of the Statistics Laboratory, University College;

${ }^{\ddagger}$ Registrar, Accident \& Emergency Unit, Regional Hospital; ${ }^{\S}$ Consultant Plastic Surgeon, Regional Hospital,

Cork, Ireland

\begin{abstract}
One of the three national games of Ireland, hurling is a contact team sport (15 a side) played with a metre long ash stick and a small hard leather ball. Over a 12 month period, 413 players were treated for hurling-related injuries at Cork Regional Hospital. While hand and facial trauma predominate, the proportion between the two sites has changed substantially from previous reports with a reduction in the level of facial injuries. The wearing of a helmet, and optionally a supplementary faceguard, is seen to have contributed to this trend. Despite this welcome reduction in facial injuries, a certain resistance to the use of protective headgear is evident, particularly among older players.

(Br J Sports Med 1995; 29 : 191-193)
\end{abstract}

Keywords: hurling; headgear; faceguard; injuries

The Irish national game of hurling has received much acclaim as both a skilful and exciting sport (Figure 1). Recognition sometimes has emanated from unexpected quarters. ${ }^{1}$

'To the outsider, hurling appears to be a fratricidal dispute over a ball (which might or might not have some relevance to the proceedings) between two sets of men armed with clubs.... eventually (one) has to admit that it takes great skill to collect and control with a single stroke of a stick a ball travelling at high speed ten feet (three metres) above the ground.'

It is perhaps not surprising that hurling-related trauma accounts for a significant proportion of all sports injuries treated in Irish hospitals. For example $18 \%$ of such cases seen at Cork Regional Hospital during 1984 involved hurling with the vast majority affecting either the hand $(33 \%)$ or face $(28 \%)^{2}$

Facial injuries have been widely reported as a potential hazard for players of stick games such as hurling, shinty and ice hockey. ${ }^{3-5}$ Perhaps not surprisingly the potential for serious eye injuries has been emphasized. ${ }^{67}$ Indeed the percentage of ice hockey players suffering eye injuries who become legally blind has been estimated as high as $16 \%{ }^{8}$

Address for correspondence: Dr P J Crowley, West Village,

Ballincollig, Co. Cork, Ireland.
Because of the various negative personal, social and financial consequences of serious injury, increasing emphasis has been placed on the need for protective measures including the wearing of appropriate headgear and a faceguard. ${ }^{25}$ While substantial progress has been made by Canadian ice hockey players with a $50 \%$ reduction in eye injuries between 1974/5 and 1983/4, the situation for hurling players is less certain with $40 \%$ of sports related facial injuries attributed to hurling in 1990. ${ }^{10}$

The current study examines the throughput of Cork Regional Hospital nine years after the original investigation. The injuries reported are assessed so as to explore a possible change in the nature of damage suffered by hurling players.

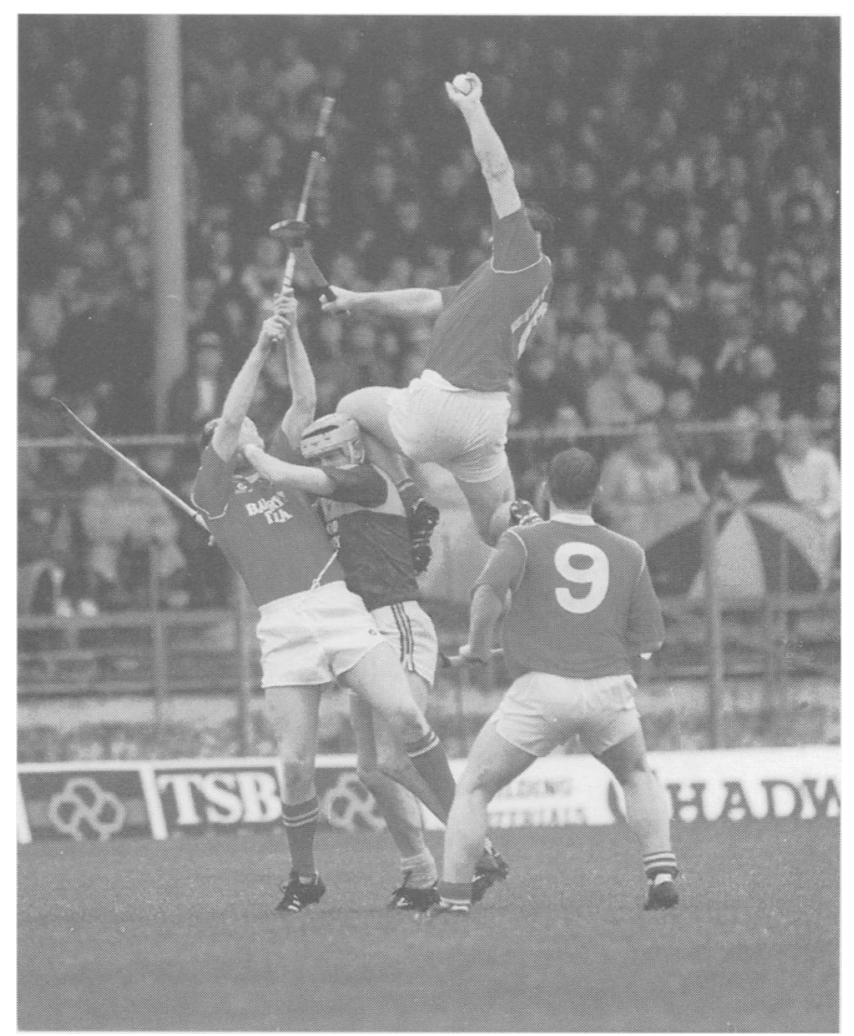

Figure 1. The sport of hurling. 


\section{Methods}

All patients treated for hurling related injuries at Cork Regional Hospital over the 12 month period from the I July, 1992 to the 30 June, 1993 were identified. Four items of information were recorded for each case. These were: (1) date of birth, (2) site of injury, (3) nature of injury, and (4) whether the player was wearing a protective helmet. When a player was wearing a helmet, it was noted whether a faceguard was also fitted.

The presentation of the results mainly involves the use of standard descriptive statistical techniques. Where appropriate statistical tests of significance are also used.

\section{Results}

A total of 413 hurling players were treated at the hospital during the study period. Age ranged from as low as 6 years up to a maximum of 44 with a mean value of 19.2 years. The very young age profile of those injured playing hurling is further emphasized if one considers the age quartiles. The youngest $25 \%$ of players range from 6 to 14 years; the next $25 \%$ of players range from 15 to 18 years; a further $25 \%$ of players between 19 and 22 years; and the oldest $25 \%$ of players range from 23 to 44 years.

Figure 2 illustrates the range of injuries suffered by the 413 players in the study. The distribution is dominated by two main sites - hand and head. Over half (56\%) of the incidents involved damage to a player's hand while a further one fifth $(20 \%)$ consisted of head injuries. These are remotely followed by ankle $(5 \%)$, knee $(5 \%)$, leg $(3 \%)$, shoulder $(3 \%)$, foot $(2 \%)$ and arm $(2 \%)$.

Information regarding usage of helmets and faceguards was noted on the hospital records for 346 ( $84 \%$ ) of the 413 patients. Approximately two out of every three players $(64 \%)$ were wearing a helmet at the time of their injury, while $44 \%$ of helmeted players also wore a faceguard.

The possibility of an age difference between users and non-users of helmets was investigated. A statistically significant difference was found with a mean age of 18.7 years for users compared to 20.4 years for non-users $(P<0.05)$. A similar pattern of younger players being more safety-conscious was seen when the helmet wearers were divided on the basis of the use or

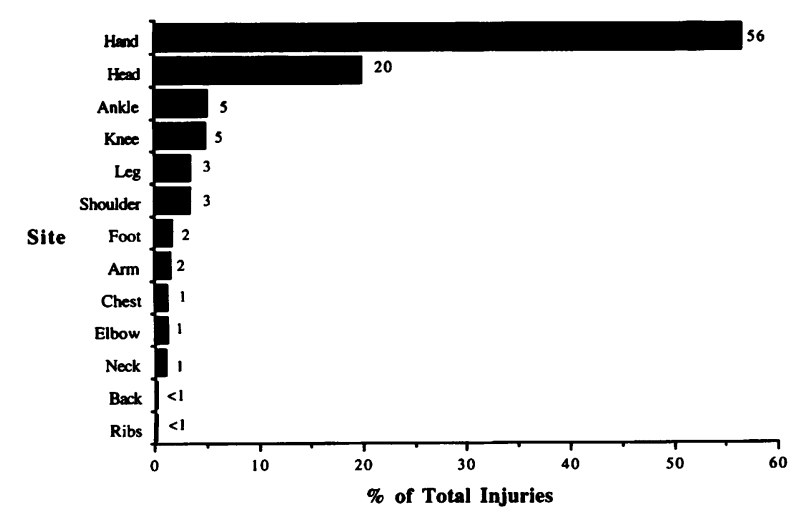

Figure 2. Profile of injuries sustained, by site.
Table 1. Summary age statistics for each site of injury

\begin{tabular}{lccc}
\hline $\begin{array}{l}\text { Site of } \\
\text { injury }\end{array}$ & Median age & Range & $\begin{array}{c}\text { Number of } \\
\text { cases }\end{array}$ \\
\hline Hand & 18 & $6-38$ & 230 \\
Head & 18 & $9-32$ & 78 \\
Ankle & 17 & $11-29$ & 21 \\
Knee & 22 & $13-44$ & 19 \\
Shoulder & 27 & $14-33$ & 14 \\
Leg & 20 & $13-34$ & 13 \\
Arm & 17 & $15-23$ & 5 \\
Chest & 25 & $18-31$ & 5 \\
Elbow & 13 & $11-19$ & 5 \\
Foot & 18 & $17-20$ & 4 \\
Neck & 17 & $15-23$ & 4 \\
Ribs & 14 & - & 1 \\
Back & 16 & - & 1 \\
\hline
\end{tabular}

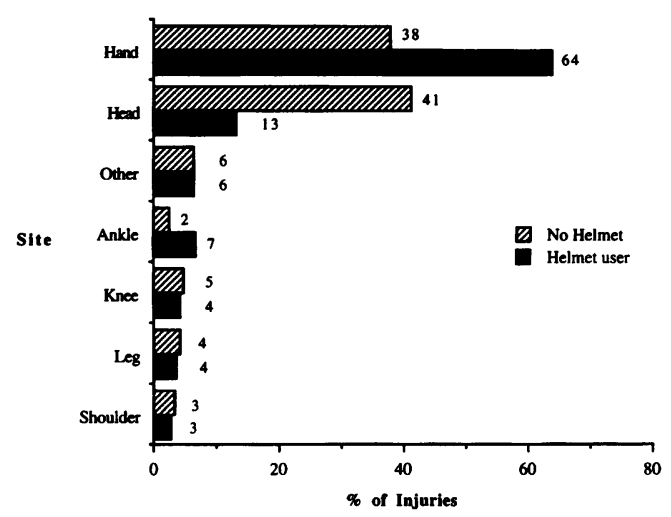

Figure 3. Comparison of site of injury between helmet users and non-users.

non-use of a faceguard; the average age of those wearing a faceguard was 17.2 years compared to 19.7 years for those without such protection $(P<0.001)$.

Summary age statistics are shown in Table 1 for the different sites of injury. While the two main categorieshand and head-have an age profile consistent with the overall group, several divergent trends are also indicated. Injuries of the shoulder, chest and knee, for example, seem more likely to affect older players while younger contestants appear more susceptible to elbow damage. One must, of course, acknowledge the small number of cases in some of these instances.

The distribution of site of injury for helmet users is compared with that for non-users in Figure 3; major differences are seen between the two groups $(P<0.001)$. The most striking finding is obviously the fact that $41 \%$ of injuries suffered by non-helmet users involved the head compared with just $13 \%$ of helmet users-more than a three to one ratio. There is some evidence that helmet users are more vulnerable to ankle injuries.

The supplementary value of a faceguard is examined in Figure 4. A further dramatic reduction in the rate of head injuries is seen $(P<0.001)$ with just $3 \%$ of players using 'helmet and faceguard' protection suffering from facial trauma compared with $21 \%$ of players using helmets only. 


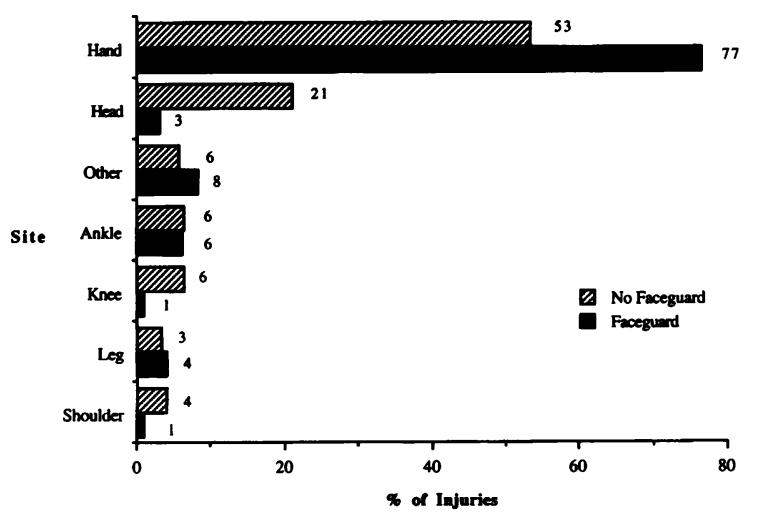

Figure 4. Comparison of site of injury between users and nonusers of faceguards.

\section{Discussion}

In recent years, hurling coaches have exerted increasing pressure on younger players to use protective headgear. The beneficial effect of this policy is clearly reflected in our results here. A continuation of the practice into older age groups is highly desirable. However, there is an undoubted tendency to discard protective measures among more experienced participants.

There are at least three ways in which increased usage of headgear might be achieved among players. The first and most drastic proposal would be to make the wearing of head protection mandatory for all players. While in theory this may be an attractive option, in practice one must take into consideration the fact that hurling is a strictly amateur sport with an associated high degree of informality and freedom regarding one's participation in the game. Consequently any attempts to impose a ruling on headgear may not receive majority support among the game's senior administrators. While player insurance considerations may lead to the introduction of compulsory headgear sometime in the future, such a possibility seems remote at this time. It is also important to note that since a high degree of skill is necessary to play the game, the actual risk of serious injury is extremely low. Thus, most participants in the game tend to ignore the possibility of significant injury. Similarly, lesser injuries are generally accepted as an inevitable consequence of one's involvement in the game.

Another potential approach to the matter could involve team coaches and mentors since they are probably in the best position to influence players. We are currently investigating variation in attitudes among hurling coaches to the overall problem of injury, and in particular to head related trauma. It is hoped that the findings of this work will provide the insights necessary to launch a successful education programme for those involved in the management of hurling teams throughout the country. Similarly, referees must be fully included in the educative process since a certain proportion of injury may be attributed to individual acts of indiscipline, indiscretion or indeed general carelessness.

The third, and probably most direct, option is to develop an effective education programme for players themselves. The extremely large number of active hurlers (estimated at c. 180000) makes this a formidable and challenging task. As a contribution towards increasing player awareness of the value of preventive measures, an illustrated chart is being prepared incorporating the main statistical findings of the current study. It is planned to distribute this chart to each hurling club and school in the country.

While particular emphasis has been placed on the potential for reducing head trauma among hurlers, it is important also to stress the importance of significantly reducing the incidence of hand injuries. The development and use of suitable gloves must be encouraged in parallel with efforts to increase the use of protective headgear. The net effect of such a policy is certain to be the continued growth and popularity of a highly skilled and unique team game.

\section{References}

1 Players bred, not made-No sport as quintessentially Irish as hurling. The Economist July 30th, 1993; 89.

2 Crowley PJ, Condon KC. Analysis of hurling and camogie injuries. Br J Sports Med 23: 183-5.

3 O'Donoghue GM, Condon KC. Facial injuries in hurlers. J Ir Med Assoc $1979 ; 72$ : 448-9.

4 Maclean JGB. A survey of shinty injuries in the Highlands during 1987-88. Br J Sports Med 23: 179-82.

5 Pashby TJ. Eye injuries in sports. J Ophthalmic Nurs Technol 1989; 8: 99-101.

6 McAuliffe-Curtin D, Cleary PE. Eye injuries due to hurling. Ir Med J 1982; 75 : 289-90.

7 Kelly S, Nolan J. Eye injuries in organised sport in rural area. $\mathrm{Br} J$ Ophthalmol 1983; 67: 837-9.

8 Pashby TJ. Eye injuries in Canadian amateur hockey. Am J Sports Med 1979; 7: 254-7.

9 Parshby TJ. Eye injuries in Canadian amateur hockey. Can J Ophthalmol 1985; 20 : 2-4.

10 Cuddihy B, Hurley M. Contact sports and injury. Ir Med J 1990; 83: $98-100$. 